Published in final edited form as:

Adv Healthc Mater. 2014 April ; 3(4): 609-616. doi:10.1002/adhm.201300151.

\title{
Rapid prototyping of concave microwells for the formation of 3D multicellular cancer aggregates for drug screening
}

\section{Ting-Yuan Tu,}

BioSystems and Micromechanics IRG, Singapore-MIT Alliance for Research and Technology (SMART) Center 1 CREATE Way, \#04-13/14 Enterprise Wing, Singapore 138602, Singapore

\section{Zhe Wang,}

BioSystems and Micromechanics IRG, Singapore-MIT Alliance for Research and Technology (SMART) Center 1 CREATE Way, \#04-13/14 Enterprise Wing, Singapore 138602, Singapore

Jing Bai,

BioSystems and Micromechanics IRG, Singapore-MIT Alliance for Research and Technology (SMART) Center 1 CREATE Way, \#04-13/14 Enterprise Wing, Singapore 138602, Singapore

\section{Wei Sun,}

BioSystems and Micromechanics IRG, Singapore-MIT Alliance for Research and Technology (SMART) Center 1 CREATE Way, \#04-13/14 Enterprise Wing, Singapore 138602, Singapore

\section{Weng Kung Peng,}

BioSystems and Micromechanics IRG, Singapore-MIT Alliance for Research and Technology (SMART) Center 1 CREATE Way, \#04-13/14 Enterprise Wing, Singapore 138602, Singapore

\section{Ruby Yun-Ju Huang,}

Cancer Science Institute of Singapore, National University of Singapore MD6, Medical Drive, Singapore 117456, Singapore

\section{Jean-Paul Thiery, and}

Institute of Molecular Cell Biology (IMCB), A-STAR Departement of Biochemistry School of Medicine, National University of Singapore Proteos, 61 Biopolis Drive, Singapore, 138673, Singapore jpthiery@imcb.a-star.edu.sg

\section{Roger D. Kamm*}

BioSystems and Micromechanics IRG, Singapore-MIT Alliance for Research and Technology (SMART) Center 1 CREATE Way, \#04-13/14 Enterprise Wing, Singapore 138602, Singapore

\footnotetext{
Abstract

Microwell technology has revolutionized many aspects of in vitro cellular studies from 2dimensional (2D) traditional cultures to 3-dimensional (3D) in vivo-like functional assays. However, existing lithography-based approaches are often costly and time-consuming. This study

Copyright WILEY-VCH Verlag GmbH \& Co. KGaA, 69469 Weinheim, Germany, 2012.

$>$ Prof. Roger D. Kamm Department of Biological Engineering and Department of Mechanical Engineering Massachusetts Institute of Technology Cambridge, MA 02139, USA. rdkamm@mit.edu.

Supporting Information

Supporting Information is available online from the Wiley Online Library or from the author.
} 
presents a rapid, low-cost prototyping method of $\mathrm{CO}_{2}$ laser ablation of a conventional untreated culture dish to create concave microwells used for generating multicellular aggregates, which can be readily available for general laboratories. Polymethylmethacrylate (PMMA), polydimethylsiloxane (PDMS), and polystyrene (PS) microwells were investigated, and each produced distinctive microwell features. Among these three materials, PS cell culture dishes produced the optimal surface smoothness and roundness. A549 lung cancer cells were grown to form cancer aggregates of controllable size from $\sim 40$ to $\sim 80 \mu \mathrm{m}$ in PS microwells. Functional assays of spheroids were performed to study migration on 2D substrates and in 3D hydrogel conditions as a step towards recapitulating the dissemination of cancer cells. Preclinical anticancer drug screening was investigated and revealed considerable differences between 2D and 3D conditions, indicating the importance of assay type as well as the utility of the present approach.

\section{Keywords}

drug screening; microwell; multicellular cancer aggregate; multicellular spheroids

\section{Introduction}

Three-dimensional (3D) tissue-like cultures are now widely used as in vitro models of in vivo cellular functions such as embryonic development, wound healing, and malignancy. Compared to a two-dimensional (2D) monolayer culture, a 3D model better mimics the in vivo tissue-like cellular features in morphology, cell-cell interaction, signal transduction and mechanical stimulation, thus providing a physiologically and pathologically relevant environment. ${ }^{[1]}$ Multicellular 3D aggregates have proven useful as a model for directing specific cell lineages using embryonic stem cell-formed embryoid bodies, ${ }^{[2]}$ and studying collective cell migration in cancer. ${ }^{[3]}$

Research on multicellular cancer spheroids (MCSs) or aggregates (MCAs) has resulted in new insights into the processes of cancer metastasis. These MCAs share common features with carcinoma in vivo and provide a better platform for studying chemo- and radioresistance of tumors. One example is the screening of therapeutic drugs via cancer cellformed MCAs. ${ }^{[4]}$ In particular, considering that cancer metastasis is initiated by cell dispersion from a solid tumor, ${ }^{[5]}$ which is a typical feature of epithelial-mesenchymal transition (EMT), ${ }^{[6]}$ recapitulating cell dispersion from multicellular aggregates provides a potentially useful in vitro model in the search for drugs that inhibit cancer cell delamination. $^{[4 \mathrm{~b}]}$

There are several established systems for culturing multicellular aggregates. A widely utilized class of methods is stationary suspension culture, e.g. non-adherent surface, spinning dish/flasks, ${ }^{[7]}$ or hanging drop methods, ${ }^{[8]}$ for ease of aggregate generation. However, the disadvantage of these methods is that a non-adherent surface fails to control aggregate size precisely. Additionally, spinning dish methods may pose detrimental levels of shear stress to delicate cell lines such as embryonic stem cells (ESCs). Hanging drop methods can grow aggregates with consistent size; however, scaling up the number of aggregates is cumbersome, and changing media without disturbing aggregates can be 
difficult. Furthermore, acquisition of time-lapse images for a particular aggregate is a challenge.

Given recent advances in microfabrication technology, microwells have become a viable alternative, providing a high-throughput method of culturing size-controllable cell aggregates. ${ }^{[9]}$ In general, a non-adhesive substrate with defined microstructure is used to isolate cells and allow them to cluster and grow into compact multicellular aggregates through cell-cell adhesion. This arrangement allows convenient observation of each aggregate and change of medium. Chips fabricated by soft lithography can also be used to control the distribution of cells and grow aggregates in a lab-on-a-chip platform. ${ }^{[10]}$

Laser fabrication offers an alternative to lithography-based approaches for microfabrication, but application of this technique to create microwells is relatively rare. ${ }^{[11]}$ Current microwell approaches based on lithographic fabrication require either chemical etching with special equipment and cleanroom facilities or a silicon master for replica molding. From an economic perspective, even minor changes in the design require a cumbersome and laborious process accompanied by a significant increase in the cost of fabrication and materials. Recent studies using 3D laser printing ${ }^{[11 \mathrm{~d}]}$ or CO laser ablation ${ }^{[12]}$ in microwells attempted to minimize fabrication complexity; however, in these studies an accessory bonding ${ }^{[12]}$ or replica molding ${ }^{[11 \mathrm{~d}]}$ process was still needed. Furthermore, irregular microwell shapes with rough structures were observed, ${ }^{[12]}$ which may potentially be harmful to cell growth.

In this work, we present a rapid and economical strategy for creating concave microwells in conventional culture dishes for generating 3D MCAs (Figure 1). To demonstrate the utility of this approach, these MCAs were used for screening anti-cancer drug compounds. We used a $\mathrm{CO}_{2}$ laser that directly "writes" microwell structures with the desired diameter and depth in less than a second (see Experimental section and Figure 1a). Microwell patterns produced by $\mathrm{CO}_{2}$ laser ablation were studied on three types of conventional substrates, namely polymethylmethacrylate (PMMA), polydimethylsiloxane (PDMS), and polystyrene (PS). Microwell diameter and depth, geometry, and surface roughness were all considered in order to identify the most suitable material for generating microwells. We found that similar size and shape of microwells can be produced under different combinations of laser parameters. To validate our microwell system for generating an in vitro 3D tumor model, the formation of MCAs using a human lung cancer cell line (A549) was characterized under different cell seeding concentrations. Functional studies were carried out to investigate the A549 MCAs in terms of migratory behaviour on 2D cell culture substrates and in 3D hydrogel conditions. As a potential application in preclinical drug screening, anti-cancer drug CI-1033 was administrated to study cellular response in A549 MCAs in 2D and 3D conditions.

\section{Results and Discussion}

\subsection{Fabrication and characterization of microwells}

The primary purpose of this study was to develop a rapid and economical microwell prototyping technique for 3D MCA generation. Performance criteria for microwell 
formation are (i) low-cost and suitability for cell growth, (ii) geometrical symmetry with sufficient volume to contain the requisite number of cells for forming aggregates, (iii) low surface roughness to prevent damage to the cell membrane.

An initial series of tests were conducted to determine the proper laser scanning speed (see Supporting Information and Figure S1). Conventional materials of PMMA, PDMS, and PS substrates were further studied and evaluated according to above criteria (Figure 2 and 3). Representative side views of microwell features (Figure 2a) show significant differences in the microwell geometry from different substrate materials. Both PMMA and PDMS microwells yielded a more conical shape with increasing laser power. PS microwells, in contrast, consistently exhibited a curved concave shape at the bottom. In addition to the dimensions $\mathrm{W}$ and $\mathrm{D}$, fabricated microwells were characterized in terms of their aspect ratio $\mathrm{AR}=\mathrm{W} / \mathrm{D}$ (Figure 2b). Results indicate that PDMS and PS microwells present similar trends in both diameter and depth, resulting in comparable AR; $\mathrm{AR}=4$ at 1 Watt and $\mathrm{AR}=$ 1 at 2-4 Watts. In contrast, PMMA shows a limited change in diameter but a major increment in depth, making the AR below 1 for almost the full range of laser power observed.

Isometric-view SEM images were examined for the three materials to elucidate its surface profile in detail (Figure 3). Microwell arrays demonstrated unique well geometry formed by each material (Figure 3a, d and g). PMMA microwells exhibited an irregular edge, debris, and micro-pores distributed over the entire internal surface (Figure 3b). The inner surface structure was confirmed through PDMS replica molding, showing elongated microwell structures with rough surfaces and high AR (Figure 3c). PDMS and PS microwells, in contrast, possessed a smooth surface (Figure $3 \mathrm{e}$ and h), and PS additionally showed axisymmetric wing structures residing at the edge of the microwell. The inner surface of PDMS revealed a cone-shaped bottom (Figure 3f). PS, on the other hand, displayed a clean and gentle concave curved bottom (Figure 3i).

These three materials revealed distinctive microwell geometry and structure after laser ablation. Pore formation on PMMA was a consequence of MMA monomer decomposition and expansion within the heated region (Figure 2c). These volatile products formed bubbles at the surface of solidified PMMA, ${ }^{[13]}$ similar to what was observed in another ablation study. ${ }^{[14]}$ The geometric difference between cone-shaped PDMS and concave PS microwells was mainly due to the different thermal diffusivity and conductivity of the materials (Figure 2f and i). ${ }^{[15]}$ Protruding structures are observed at the edge of each PS microwell, which can be attributed to the gradient in surface tension of PS caused by the temperature gradient created during the local laser heating process. ${ }^{[16]}$ The laser beam was slightly elliptical in shape and therefore created an asymmetric heat-affected zone as it evaporated the PS material. The surface-tension-driven force resulted in a slightly non-axisymmetric accumulation of molten liquid PS and formed a recast region after resolidification (Figure 2h). ${ }^{[17]}$ Since a majority of cells are grown in the middle of the concave zone, recast areas residing at the sides of PS microwells should rarely cause any significant effect on cell growth. The ability to observe cells was adversely affected in PDMS microwells due to their cone-shaped bottom (Figure 3f). PS microwells, with a gentle curvature at the bottom, were considered to be much more favorable for monitoring cells in real-time (Figure 3i). 
Moreover, curved microwell structures have been reported to promote the formation of aggregates of more uniform size and shape. ${ }^{[9 b]}$

Our findings suggest that among the three most commonly used cell culture materials, PS is preferred in terms of its material properties, microwell structure and morphology. In addition, PS is the most commonly used material in tissue and cell culture research, [11a, 18] and has previously been used for thermal molding by a $\mathrm{CO}_{2}$ laser for various biological applications. ${ }^{[11 a]}$ The PS chosen in this study is from untreated cell culture dishes, which are intended for growing cells in stationary suspension, and are therefore both economical and readily available.

\subsection{Generation of MCAs}

A549 lung cancer cells were seeded in the 100 x 100 array of PS microwells in an untreated cell culture dish to test their ability for MCA formation. The process of cell aggregation was directly observed from d1, the day of seeding cells, to d5 (Figure 4a-c). On d1, 20 cells were observed in each microwell, and the morphology and distribution of individual cells and cell aggregates were observed (Figure 4a). Due to the confinement by the microwell, cells formed loose cluster. From d1 to d4, cells were observed to gradually maximize adhesion with other cells to form a loose aggregate, with increasingly blurred cell-cell interfaces (Figure 4b). Compact MCA could be observed in d4 or d5 (Figure 4c). Surface topography of MCAs at d5 was characterized using SEM (Figure 4d and e). Results showed that cells compactly associated with each other, forming an MCA as an integral in vivo-like tissue. Retrieved MCAs were also stained with live cell fluorescent marker to test their viability. Results suggested that the MCAs remained viable after culturing in the microwells for 5 days (Figure 4f).

Our data demonstrate that PS microwells are capable of growing size-controllable 3D MCA using an A549 lung cancer cell line. We have tested different cell lines in our PS microwell, e.g. bladder cancer cells, breast cancer cells and embryonic carcinoma, revealing reliable MCA formation (data not shown). On an untreated surface that does not facilitate cell adhesion, cells preferentially favor cell-cell adhesion and form smooth aggregates (Figure 4a- c). ${ }^{[19]}$ The specific morphology of the resulting MCAs varies with cell number, microwell dimension, and different cell lines ${ }^{[4]}$. The A549 line used in this study forms MCAs (below $\sim 20$ cells per microwell) that are not smoothly spherical but remain intact during subsequent manipulation (Figure 4d-f). ${ }^{[20]}$

Other factors influencing the diameter of MCAs were examined using the PS concave microwells of width $117 \mu \mathrm{m}\left(\mathrm{M}_{1}\right.$ microwell $)$ and $229 \mu \mathrm{m}\left(\mathrm{M}_{2}\right.$ microwell $)$ corresponding to laser powers of $2 \mathrm{~W}$ and $5 \mathrm{~W}$, respectively, with the cell seeding concentrations of 1,5 , and $10 \times 10^{4} \mathrm{cells} / \mathrm{ml}$ (Figure $4 \mathrm{~g}$ and $\mathrm{h}$ ). Results showed that MCA diameter increased with increasing cell seeding density in $\mathrm{M}_{2}$ microwells, spanning from $\sim 60 \mu \mathrm{m}$ to $\sim 80 \mu \mathrm{m}$ (Figure $4 \mathrm{~g}$ ); in contrast, the $\mathrm{M}_{1}$ microwells produced MCAs of diameter $\sim 40 \mu \mathrm{m}$ for all three seeding densities. The probability of MCAs forming at different cell seeding concentrations was also compared (Figure $4 \mathrm{~h}$ ). $\mathrm{M}_{2}$ microwells demonstrated a consistent $\sim 80$ to $90 \%$ probability of forming MCAs for all seeding densities (1,5 and $10 \times 10^{4}$ cells $\left./ \mathrm{ml}\right)$. For the $\mathrm{M}_{1}$ microwells, in contrast, the probability of MCA formation varied with seeding density. 
Those microwells lacking an MCA contained either no cells or only dead cells (data not shown).

The different MCA populations between $\mathrm{M}_{1}$ and $\mathrm{M}_{2}$ microwells may be partially explained by the different number of cells trapped in the microwell. Immediately after cell seeding, $\mathrm{M}_{1}$ microwells typically contained $\sim 5$ to $\sim 8$ cells, while $\mathrm{M}_{2}$ wells contained $\sim 10$ to $\sim 20$ cells. Another possible explanation is that both types of microwell were fabricated in a $100 \times 100$ grid with $0.5 \mathrm{~mm}$ spacing, resulting in a lower fraction of total surface covered for $\mathrm{M}_{1}$ microwells (Figure S2), such that more cells would settle in the empty space between $\mathrm{M}_{1}$ wells without seeding in the microwells. $\mathrm{M}_{1}$ microwells were also found to have lower probability of forming MCAs (Figure 4h). This result can be explained by noting that $\mathrm{M}_{1}$ microwells were not only smaller in width but also in depth in a given $A R=1$. For this reason, during medium change or when transferring the microwell plate from incubator to cell culture hood, more cells may have been disturbed and displaced from the microwell. Such a situation could be compensated for by using a higher cell seeding density or a more closely packed microwell pattern. The present findings (Figure $4 \mathrm{~g}$ and $\mathrm{h}$ ) may help to guide the choice of a particular size of microwell to obtain the desired size of MCA with sufficient frequency of its formation. For instance, to make $\sim 40 \mu \mathrm{m}$ MCAs, $\mathrm{M}_{1}$ microwells at a cell concentration of $10 \times 10^{4}$ cells $/ \mathrm{ml}$ would be an appropriate choice to ensure high probability of MCA formation, while for $\sim 80 \mu \mathrm{m}$ MCAs we may choose $\mathrm{M}_{2}$ microwells with a seeding concentration of $10 \times 10^{4}$ cells $/ \mathrm{ml}$. The results can serve as a reference to optimize sizecontrollable MCA formation in the future.

\subsection{MCA migration assay}

To demonstrate the functionality of the formed MCAs, we chose to study two typical applications, cell migration and drug screening. Cell dispersion from aggregates and subsequent migration play a pivotal role in a variety of physiological and pathological processes. The dissemination of cancer cells from a solid tumor is an essential step for invasion and metastasis of tumors. Moreover, cells preferentially leverage different mechanisms in migrating in a $2 \mathrm{D}$ or $3 \mathrm{D}$ environment. ${ }^{[21]}$ Therefore, functional studies of cancer cell migration could potentially assist in the understanding of such invasion process. To explore this, we studied cell migration both on a 2D substrate and in a collagen-based ECM 3D environment.

In the 2D migration assay, A549 MCAs were seeded on a polystyrene cell culture substrate and imaged from 2 - $24 \mathrm{~h}$ (Figure 5a). Migration was initiated by cells attached to the substrate, defined as a "precursor film". ${ }^{[22]}$ Tracking the motion of each cell revealed that the MCAs consistently spread in a radial fashion (Figure 5b). During the process of A549 MCA spreading, the precursor film maintained cell-cell contact and migrated collectively for 8 - $10 \mathrm{~h}$. Large filopodia were observed at the front film edge (Figure $5 \mathrm{c}$ ). Collective migration was gradually superseded by individual cell mobility as cells began to escape from the outer edge (Figure 5d).

To examine the kinetics of evolution of the monolayer, we pursued an analytical approach similar to that described by Douezan et al. to quantify MCA spreading on a planar substrate by measuring spreading area while cells migrated in the collective mode during the first $10 \mathrm{~h}$ 
(Figure S3). ${ }^{[22]}$ Spreading area of the precursor film at each time point is proportional to the initial size of the MCA, in that larger aggregates spread faster than smaller ones (Figure S3a). We also examined the influence of EGF addition on the precursor film. As expected, results exhibit faster migration with added EGF independent of aggregate size (Figure S3b).

Previous work has shown that the cell aggregate spreading on a 2D planar substrate can be described as a competition of cell-cell and cell-substrate adhesion. ${ }^{[23]}$ Douezan et al. further demonstrated that the aggregate spreading process resembles a liquid-gas transition that depends on the cohesiveness of the aggregate. ${ }^{[22]}$ Our results further support that this transition occurs consistently with varied MCA size but varies with cohesion strength (Figure S3b). One explanation for this result is that MCA cohesiveness was altered due to the addition of EGF. EGF is known to promote E-cadherin endocytosis, ${ }^{[24]}$ as well as to repress E-cadherin transcription in a short time. ${ }^{[25]}$ The loss of E-cadherin resulted in reduction of cell-cell adhesion energy, which preferentially caused aggregates to dissociate.

In addition to the 2D assay, migration in 3D was observed by embedding A549 MCAs in native type I collagen (Figure 5e). Migration in 3D was confirmed by observing their trajectories and morphologies over $24 \mathrm{~h}$ (Figure 5f). In the first 2 - $4 \mathrm{~h}$, some protrusions extended into the gel matrix (indicated with arrows) to present at the surface of the MCA (Figure $5 \mathrm{~g}$ ). After $4 \mathrm{~h}$, cells in the aggregate started to move, subsequently causing a rearrangement in MCA shape. Elongated multicellular protrusions later formed as the cells migrated outwards. These pseudopodia- or invadopodia-like protrusions from the MCAs are actin-rich structures important in cancer cell migration and invasion. ${ }^{[26]}$ In most cases, the MCA then broke into two or three multicellular pieces, and individual cells also began to escape from the aggregate (Figure $5 \mathrm{~h}$ ), showing that cells depend on different mechanisms when migrating in the matrix. ${ }^{[27]}$

\subsection{Screening of migration-inhibition drug}

Despite the distinct functional behavior of 2D and 3D migration on MCAs, studies have also demonstrated that MCAs showed dramatically different responses to drug treatment ${ }^{\text {[9a, 20a] }}$ To demonstrate the ability to screen drugs according to their effect on cell migration, we treated MCAs with EGF and tested the inhibitory activity of EGFR tyrosine kinase antagonist. CI-1033, a pan-ErbB tyrosine kinase inhibitor, has been reported in a number of Phase I and Phase II clinical trials in patients with non-small cell lung cancer. ${ }^{[28]}$ The influence of CI-1033 on MCA dispersion was investigated under the two conditions described above. Using as a baseline for comparison the condition where only EGF was applied, the change of cell dispersion index $\Delta$ (see Supporting Information and Equation S8), a measure of the size of the region of cell spreading, was used as an indicator of the effectiveness of the drug. For different drug concentrations applied in the two conditions, normalized dispersion (Equation S9) was plotted against time (Figure 6).

Both 2D and 3D migration increased with time except in cases of complete inhibition of dispersion for high doses of CI-1033. The growth rate of cell dispersion is inversely correlated to the dimensionality of the environment and the dose of CI-1033. Cells in 2D migrated more than cells in 3D at all concentrations of drug (Figure 6a). Also, full inhibition of cell migration in 2D conditions required a $\sim 50 \mu \mathrm{M}$ dose of CI-1033, two orders of 
magnitude higher than the dose of $\sim 0.5 \mu \mathrm{M}$ needed to inhibit migration in 3D (Figure $6 \mathrm{~b}$ ). Data at the 24-h endpoint are plotted against the dosage to obtain a dose-response curve (Figure 7), and to determine the effective dose of CI-1033 for dispersion inhibition. Curve fitting results revealed that the IC50 value dropped from 3,000 $\mathrm{nM}$ in 2D to $30 \mathrm{nM}$ in 3D. Results suggest that preclinical drug screening could be applied by using this 3D MCA model.

The 2D dispersion assay relies on different mechanisms for cell migration and differs considerably from the 3D assay in terms of A549 MCA response. As a result of a competition between cell-cell and cell-substrate adhesion, MCA 2D migration was more strongly affected by MCA size and choice of substrate. These factors showed less effect on MCAs encapsulated by ECM in the 3D assay, where matrix metalloproteinases (MMPs) for ECM remodeling are known to be an important factor in addition to the strength of adhesion and cohesion. Our data show that compared to a $2 \mathrm{D}$ environment, the concentration required for inhibition of cell dispersion, as reflected by the IC50, fell by a factor of nearly 100 in 3D conditions (Figure 7). In contrast, in a different experimental setup studying cytotoxic drug resistance, A549 MCAs showed a ten to hundred fold change in resistance than that of monoculture. ${ }^{[20 a]}$ These results underscore the importance of different assay types for MCAs as well as the practicability of our platform for future drug screening applications.

Despite the many advantages of our present approach, some limitations should be addressed: (i) The size of the microwell is confined to $\sim 100-400 \mu \mathrm{m}$ due to the laser beam size. (ii) Thermal processing created a recast zone that resides on the surface area and reduces the number of PS microwells that can be created on a given surface area. (iii) MCAs must be retrieved manually, a problem common to other microwell methods.

The PS microwell dish presented is manufactured directly by patterning with a desktop $\mathrm{CO}_{2}$ laser, requiring no additional fabrication processes. Drilling a microwell requires less than $500 \mathrm{~ms}$, and a pattern of 1,000 microwells can be generated in $2.5 \mathrm{~min}$. Our approach of manufacturing a microwell array from a Petri dish facilitates liquid handling and ensures ease of material access suitable for most biological and medical laboratories. The PS dishes that we used in this study could be reused as many as five to seven times. This microwell technique can potentially be integrated with a microfluidic system. Using this approach, medium and reagent could be exchanged rapidly and conveniently. Potential drug screening assays may be performed within the microfluidic setup, eliminating the need to retrieve aggregates from the wells. The small channel volume of microfluidic devices could allow minimal consumption of expensive compounds. With proper design of fluidic compartments, individual environment conditions could be created for each MCA in a microwell to attain high-throughout screening.

\section{Conclusions}

We have presented a simple, economical and high-throughput method for concave microwell prototyping on a conventional culture dish. Microwells generated by $\mathrm{CO}_{2}$ laser ablation on PMMA, PDMS and PS materials were investigated. PS revealed the best surface smoothness and roundness among these three materials. A549 MCAs were successfully 
generated in the PS microwell, and aggregate size could be controlled. MCA migration was examined in 2D and 3D conditions showing distinctive migration patterns due to the environmental dimensionality. Pre-clinical therapeutic anti-cancer drug screening was performed to identify the effective dosage for $2 \mathrm{D}$ and $3 \mathrm{D}$ conditions. This technique has potential applications in generating 3D multicellular aggregates for both basic and translational research in the future.

\section{Experimental section}

\section{Microwell prototyping}

Microwell prototyping was implemented using a commercial air-cooled $10.6 \mu \mathrm{m} \mathrm{CO} 2$ laser engraving/cutting system (VLS-2.30, Universal Laser System Inc., U.S.A.) with high power density focusing optics and maximum output power of $10 \mathrm{~W}$. The focused laser beam was operated in TEM00 mode, ablating the material surface to directly pattern the substrate of interest. Laser energy was capable of heating all tested surfaces sufficiently to melt and gasify. The melted surface was purged by laser pressure in a Gaussian distribution producing a lip around the circumference of the ablated zone and a concave well-like space in the middle suitable for cell docking (Figure 1a). Wells were characterized by their top opening width $\mathrm{W}$ and top-to-bottom depth, D.

Multi-well patterns were designed using commercial software (AutoCAD 2007, Autodesk Inc.) and transferred to the laser system. Patterns used for the present study contained $100 \mathrm{x}$ 100 microwell arrays spaced $0.5 \mathrm{~mm}$ by $0.5 \mathrm{~mm}$. Laser scanning was performed by a XY mobile Focus Carriage (FC) travelling to the location of interest (Figure1b). The characteristics of these microwells were assessed with the combinations of laser power and scanning speed on PS material. With a desired laser output, microwells were generated on PMMA (1 mm thick), PDMS ( $2 \mathrm{~mm}$ thick, Sylgard 184, Dow Corning), and PS (1 mm thick, $90 \mathrm{~mm}$ untreated tissue culture dish, VWR). Microwells were washed with 70\% ethanol for both disinfection and bubble removal, and then washed twice with PBS to replace the ethanol and to clean debris from laser fabrication. The fabricated PS microwells were preserved in PBS solution before use.

\section{Cancer cell culture}

A549 human lung adenocarcinoma epithelial cells transfected with histone H2B-mCherry cDNA for nuclear staining were cultured in a T75 flask (Nunclon ${ }^{\mathrm{TM}} \Delta$ Surface) in Dulbecco's modified Eagle medium (Gibco/Invitrogen 12100) supplemented with $10 \%$ fetal bovine serum (Gibco/Invitrogen), $1 \%$ penicillin-streptomycin. Puromycin $0.01 \%$ was added for the selection of mCherry - expressing cells, along with the medium change every $36 \mathrm{~h}$ to $48 \mathrm{~h}$. For passage, cells at $80-90 \%$ confluency were detached from the culture flask by treating with $0.05 \%$ trypsin-ethylenedinitriletetraacetic acid (Trypsin-EDTA) at $37{ }^{\circ} \mathrm{C}$ in an incubator for $3 \mathrm{~min}$. Medium was then added to inhibit the enzymatic reaction of TrypsinEDTA. Sub-culture seeding density was kept at 2-3 x $10^{5}$ cells/ T75 flask. 


\section{Generation of MCAs}

PS microwells were coated with a layer of anti-adhesive repellent using PBS with $0.2 \%$ pluronic (Pluronic F108, BASF) for $1 \mathrm{~h}$ (Figure 1c). ${ }^{[29]}$ Subsequently, the coated microwell plate was washed twice with PBS to minimize free polymers in the plates. ${ }^{[30]}$ The pluronic coating has been shown to stably repel cell attachment for $\sim 1$ week during cell culture incubation. ${ }^{[31]}$ Cell suspensions, detached using the aforementioned procedure for passage, were mixed with $10 \mathrm{ml}$ culture medium and then added into the dish. After incubation for 1 $\mathrm{h}$, cells that had not embedded inside each microwell were removed by exchanging with 10 $\mathrm{ml}$ of fresh medium (Figure 1d). ${ }^{[9 \mathrm{~b}]}$ Culture medium containing $0.01 \%$ puromycin was changed every $4 \mathrm{~d}$. Cells embedded in each microwell would form aggregates through cellcell and cell-matrix adhesion (Figure 1e). Multiple MCAs were generated from a conventional culture dish comprising a microwell array (Figure 1f).

\section{Scanning electron microscopy}

Surface structures of both microwells and MCAs were investigated using scanning electron microscopy (SEM) (JEOL Ltd., JSM-6510LV). Prior to SEM imaging of the MCAs, medium was aspirated and rinsed with PBS. MCAs were fixed using $0.1 \mathrm{M} \mathrm{Na-Cacodylate}$ buffer, $2.0 \%$ paraformaldehyde, and $2.5 \%$ glutaraldehyde applied for $4 \mathrm{~h}$ at room temperature, then rinsed with $0.1 \mathrm{M} \mathrm{Na}$-Cacodylate buffer on a shaker table for $10 \mathrm{~min}$. Post-fixation was done using $1.0 \%$ aqueous osmium tetroxide in $0.1 \mathrm{M} \mathrm{Na-Cacodylate}$ buffer in a dark fume hood for $90 \mathrm{~min}$, and followed by rinsing with $0.1 \mathrm{M} \mathrm{Na-Cacodylate}$ buffer on a shaker table for another $10 \mathrm{~min}$. Finally, samples were serially dehydrated by different concentrations of ethanol $(37 \%, 67 \%, 95 \%$, and $100 \%)$ on a shaker table for 10 min each, followed by critical point drying (Balzers, BALSERS CPD 030) and platinum sputtered in vacuum (JEOL Ltd., JFC-1600).

\section{D and 3D drug screening}

A549 MCAs $\sim 60$ [.proportional]m in diameter were retrieved from the microwell dish. 10-20 MCAs were seeded in individual wells of a 96-well plate, for both 2D and 3D assays. For 3D assays, $200 \mu \mathrm{l}$ collagen gel solution at a concentration of $2.5 \mathrm{mg} / \mathrm{ml}$ and $\mathrm{pH}$ 7.4 was prepared on ice with $123.8 \mu \mathrm{l}$ type I collagen ( $4.04 \mathrm{mg} / \mathrm{ml}$, BD Bioscience) mixed with $29.7 \mu \mathrm{l}$ cell culture water, $20 \mu \mathrm{l}$ 10X PBS, 6.5[.proportional]1 $\mathrm{NaOH}$, and $20 \mu \mathrm{l}$ medium with MCAs. ${ }^{[32]}$ MCAs were supplemented with DMEM medium that contained an additional $20 \mathrm{ng} / \mathrm{ml}$ epidermal growth factor (EGF), and for a range of CI-1033 concentrations: $0.1,0.5,1,5,10,50,100,500,1,000,5,000,10,000,50,000$, and 100,000 $\mathrm{nM}$.

\section{Image acquisition and analysis}

3D image stacks of each MCA were acquired using confocal microscopy (Fluoview 1000, Olympus) with a $10 \mathrm{X}$ objective (NA $=0.4$ ) to take hourly images of cell dispersions over a $24 \mathrm{~h}$ period. Quantitative analysis of MCA dispersion was accomplished by image analysis using Imaris software (Bitplane). Segmentation and calculation are described in detail in supporting information. 


\section{Acknowledgments}

This research was supported by the National Research Foundation Singapore through the Singapore MIT Alliance for Research and Technology's [BioSyM IRG] research program and Mechanobiology Institute at National University of Singapore. The authors would like to thank Dr. Chang-Yu Chen, Institute of Applied Mechanics at National Taiwan University for providing constructive advice in laser fabrication.

\section{Abbreviations}

$\begin{array}{ll}\text { 2D } & \text { 2-dimensional } \\ \text { 3D } & \text { 3-dimensional } \\ \text { ECM } & \text { Extracellular matrix } \\ \text { EGF } & \text { Epidermal growth factor } \\ \text { EMT } & \text { Epithelial-mesenchymal transition } \\ \text { MCS } & \text { Multicellular cancer spheroids } \\ \text { MCA } & \text { Multicellular cancer aggregates } \\ \text { PDMS } & \text { Polydimethylsiloxane } \\ \text { PMMA } & \text { Polymethylmethacrylate } \\ \text { PS } & \text { Polystyrene }\end{array}$

\section{References}

1. a Wendt D, Riboldi SA, Cioffi M, Martin I. Adv. Mater. 2009; 21:3352. [PubMed: 20882502] b Wolf K, Wu YI, Liu Y, Geiger J, Tam E, Overall C, Stack MS, Friedl P. Nat. Cell Biol. 2007; 9:893. [PubMed: 17618273]

2. Hwang Y, Chung BG, Ortmann D, Hattori N, Moeller H, Khademhosseini A. Proc. Natl. Acad. Sci. U. S. A. 2009; 106:16978. [PubMed: 19805103]

3. Friedl P, Gilmour D. Nat. Rev. Mol. Cell Biol. 2009; 10:445. [PubMed: 19546857]

4. a Hakanson M, Textor M, Charnley M. Integr. Biol. 2011; 3:31.b Aref AR, Huang RY, Yu W, Chua KN, Sun W, Tu TY, Bai J, Sim WJ, Zervantonakis IK, Thiery JP, Kamm RD. Integr. Biol. 2013; 5:381.

5. Chambers AF, Groom AC, MacDonald IC. Nat. Rev. Cancer. 2002; 2:563. [PubMed: 12154349]

6. a Thiery JP. Nat. Rev. Cancer. 2002; 2:442. [PubMed: 12189386] b Thiery J, Acloque H, Huang R, Nieto M. Cell. 2009; 139:871. [PubMed: 19945376]

7. Castaneda F, Kinne RK, Cancer Res J. Clin. Oncol. 2000; 126:305.

8. Tung YC, Hsiao AY, Allen SG, Torisawa YS, Ho M, Takayama S. Analyst. 2011; 136:473. [PubMed: 20967331]

9. a Kloss D, Fischer M, Rothermel A, Simon J, Robitzki A. Lab Chip. 2008; 8:879. [PubMed: 18497906] b Choi YY, Chung BG, Lee DH, Khademhosseini A, Kim J, Lee S. Biomaterials. 2010; 31:4296. [PubMed: 20206991] c Zorlutuna P, Annabi N, Camci-Unal G, Nikkhah M, Cha JM, Nichol JW, Manbachi A, Bae H, Chen S, Khademhosseini A. Adv. Mater. 2012; 24:1782. [PubMed: 22410857]

10. a Ong SM, Zhang C, Toh YC, Kim SH, Foo HL, Tan CH, van Noort D, Park S, Yu H. Biomaterials. 2008; 29:3237. [PubMed: 18455231] b Kim C, Lee K, Bang J, Kim Y, Kim M, Oh K, Lee S, Kang J. Lab Chip. 2011c Wlodkowic D, Faley S, Skommer J, McGuinness D, Cooper JM. Anal Chem. 2009; 81:9828. [PubMed: 19902928] d Wlodkowic D, Faley S, Zagnoni M, Wikswo JP, Cooper JM. Anal Chem. 2009; 81:5517. [PubMed: 19514700] e Wlodkowic D, 
Skommer J, McGuinness D, Faley S, Kolch W, Darzynkiewicz Z, Cooper JM. Anal Chem. 2009; 81:6952. [PubMed: 19572560]

11. a Young EW, Berthier E, Guckenberger DJ, Sackmann E, Lamers C, Meyvantsson I, Huttenlocher A, Beebe DJ. Anal. Chem. 2011; 83:1408. [PubMed: 21261280] b Chen C, Liu K, Jong D, Wo A. Appl. Phys. Lett. 2007; 91:123901.c Chen C, Tu T, Jong D, Wo AM. Lab Chip. 2009; 9:2370. [PubMed: 19636469] d Napolitano AP, Chai P, Dean DM, Morgan JR. Tissue Eng. 2007; 13:2087. [PubMed: 17518713]

12. Selimovic S, Piraino F, Bae H, Rasponi M, Redaelli A, Khademhosseini A. Lab Chip. 2011; 11:2325. [PubMed: 21614380]

13. Hertzberg M, Zlochower IA. Combust. Flame. 1991; 84:15.

14. Nayak NC, Lam YC, Yue CY, Sinha AT. J. Micromech. Microeng. 2008; 18:095020.

15. Liu H, Gong H. J. Micromech. Microeng. 2009:1.

16. Wu S. J. Phys. Chem. 1970; 74:632.

17. Chen SC, Cahill DG, Grigoropoulos CP, Heat Transfer J. 2000:122.

18. Khan F, Zhang R, Unciti-Broceta A, Díaz-Mochón JJ, Bradley M. Adv. Mater. 2007; 19:3524.

19. Liu J, Abate W, Xu J, Corry D, Kaul B, Jackson SK. Innate Immun. 2011; 17:245. [PubMed: 20418262]

20. a Desoize B, Jardillier J. Crit Rev Oncol Hematol. 2000; 36:193. [PubMed: 11033306] b Liu J, Abate W, Xu J, Corry D, Kaul B, Jackson SK. Innate Immun. 2011; 17:245. [PubMed: 20418262]

21. Meyer AS, Hughes-Alford SK, Kay JE, Castillo A, Wells A, Gertler FB, Lauffenburger DA. J Cell Biol. 2012; 197:721. [PubMed: 22665521]

22. Douezan S, Guevorkian K, Naouar R, Dufour S, Cuvelier D, Brochard-Wyart F. Proc. Natl. Acad. Sci. U. S. A. 2011; 108:7315. [PubMed: 21504944]

23. Ryan PL, Foty RA, Kohn J, Steinberg MS. Proc. Natl. Acad. Sci. U. S. A. 2001; 98:4323. [PubMed: 11274361]

24. Lu Z, Ghosh S, Wang Z, Hunter T. Cancer Cell. 2003; 4:499. [PubMed: 14706341]

25. Lee MY, Chou CY, Tang MJ, Shen MR. Clin. Cancer Res. 2008; 14:4743. [PubMed: 18676743]

26. Shankar J, Messenberg A, Chan J, Underhill TM, Foster LJ, Nabi IR. Cancer Res. 2010; 70:3780. [PubMed: 20388789]

27. a Sabeh F, Shimizu-Hirota R, Weiss SJ. J. Cell. Biol. 2009; 185:11. [PubMed: 19332889] b Li XY, Ota I, Yana I, Sabeh F, Weiss SJ. Mol. Biol. Cell. 2008; 19:3221. [PubMed: 18495869]

28. a Janne PA, von Pawel J, Cohen RB, Crino L, Butts CA, Olson SS, Eiseman IA, Chiappori AA, Yeap BY, Lenehan PF, Dasse K, Sheeran M, Bonomi PD. J. Clin. Oncol. 2007; 25:3936. [PubMed: 17761977] b Zinner RG, Nemunaitis J, Eiseman I, Shin HJ, Olson SC, Christensen J, Huang X, Lenehan PF, Donato NJ, Shin DM. Clin. Cancer Res. 2007; 13:3006. [PubMed: 17505003]

29. a Kuo CT, Chiang CL, Huang RYJ, Lee H, Wo AM. NPG Asia Mater. 2012:4.b Liu VA, Jastromb WE, Bhatia SN. J Biomed Mater Res. 2002; 60:126. [PubMed: 11835168]

30. Wong I, Ho CM. Microfluid Nanofluidics. 2009; 7:291. [PubMed: 20357909]

31. Corey JM, Gertz CC, Sutton TJ, Chen Q, Mycek KB, Wang BS, Martin AA, Johnson SL, Feldman EL. J. Biomed. Mater. Res., Part A. 2010; 93:673.

32. Shin Y, Han S, Jeon JS, Yamamoto K, Zervantonakis IK, Sudo R, Kamm RD, Chung S. Nat. Protoc. 2012; 7:1247. [PubMed: 22678430] 


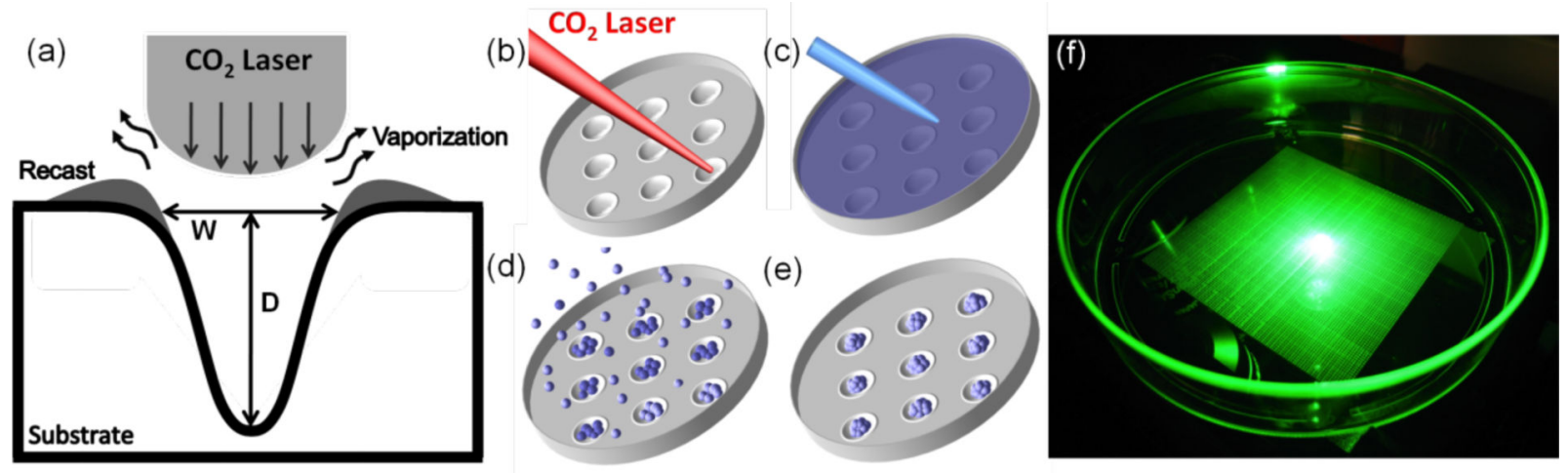

Figure 1.

Schematic diagram of the microwell fabrication process for generation of 3D aggregates (a) Illustration of laser ablation process. Substrate is melted by $\mathrm{CO}_{2}$ laser energy distributed in a Gaussian profile. The ablation leaves a concave well with a recast zone at its edge. (b) Laser drilling via an XY mobile focus carriage travelling to the location of interest. (c) A thin layer of pluronic was deposited to prevent cell attachment on the substrate. (d) Cell seeding. (e) Formation of aggregates after 4-5 days. (f) A snapshot of microwell array pattern in a cell culture dish. 
(a)
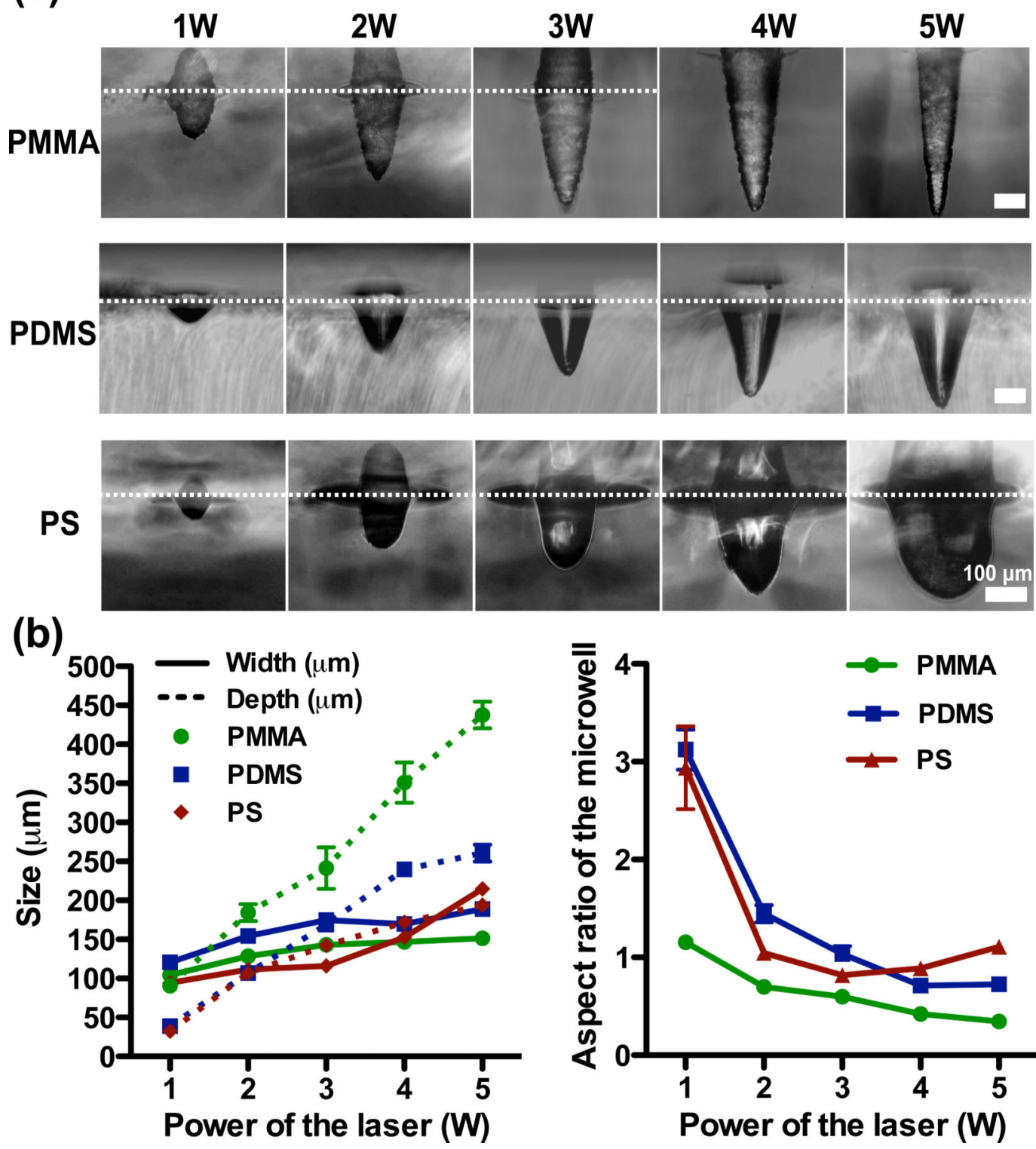

(b)

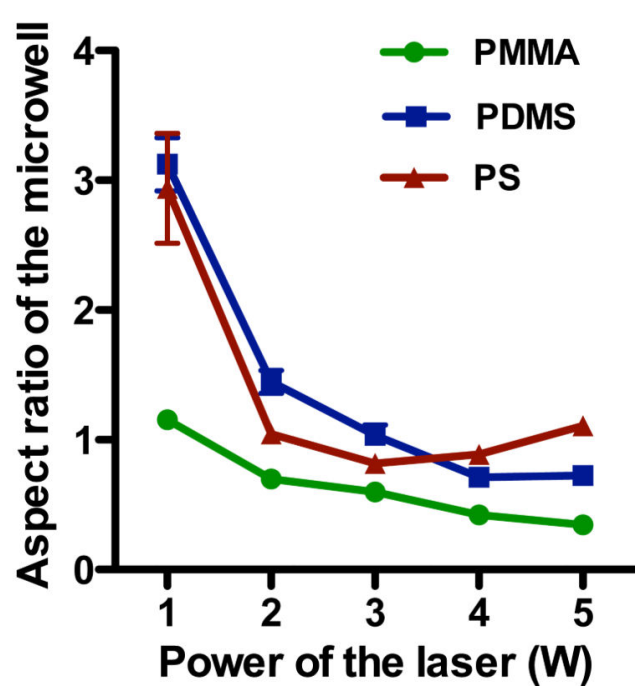

Figure 2.

Characterization of microwells on PMMA, PDMS, and PS. (a) Representative microwell features with respect to a range of laser power from $1 \mathrm{~W}-5 \mathrm{~W}$. For clarity, the reflection plane is indicated in white dotted line. (b) Evaluation of diameter, depth, and aspect ratio of microwell in different materials. 

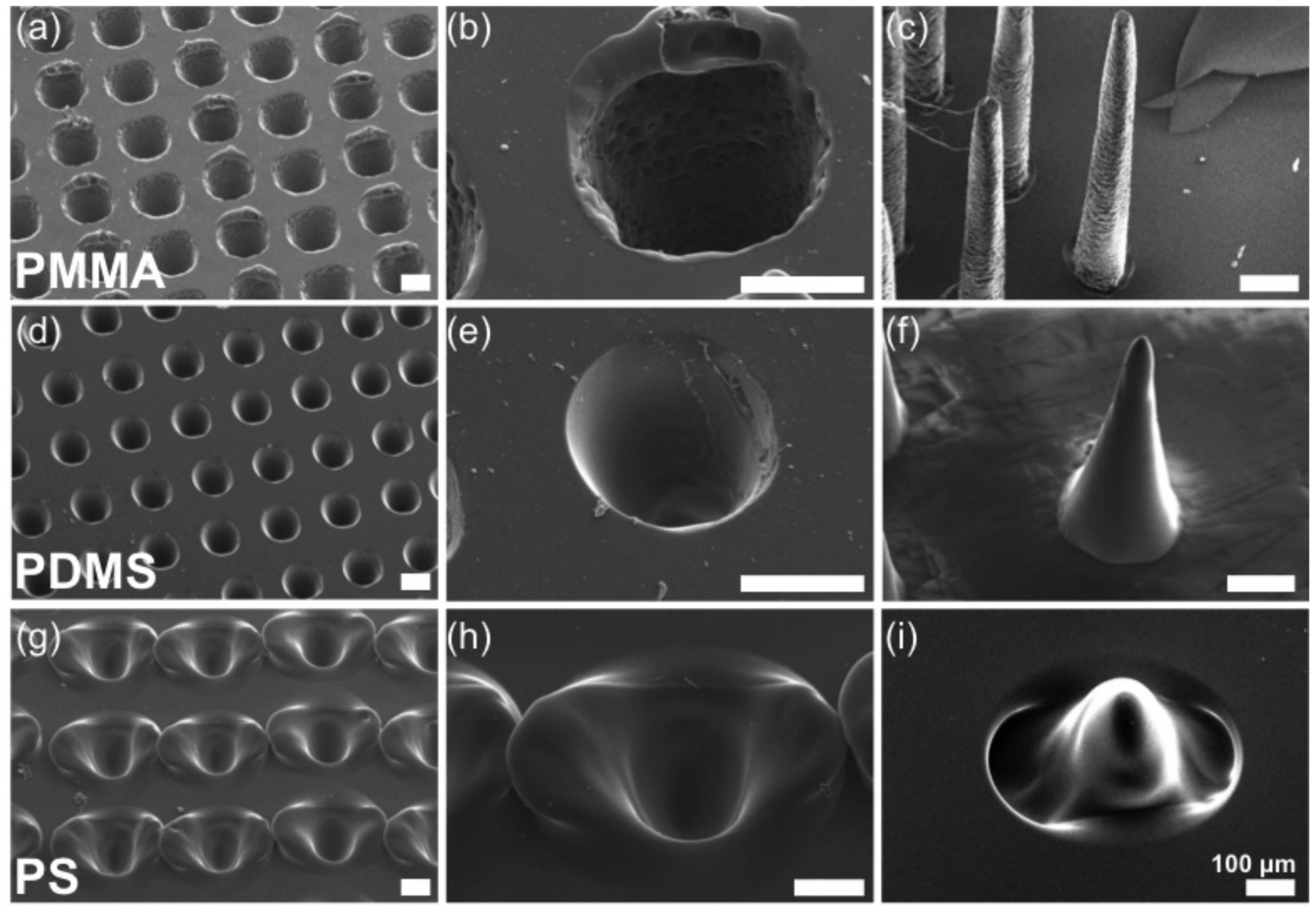

Figure 3.

Isometric-view SEM images of microwells from three materials. A detailed surface profile of a single microwell is presented (middle), along with a negative formed by PDMS replica molding (right). (a)-(c) PMMA microwells exhibit a rough surface at the periphery and have micro- pores distributed over much of the internal surface. (d)-(f) PDMS microwells exhibit a smooth surface and a cone-shaped bottom. (g)-(i) PS microwells have smooth surface characteristics with substantial recast material around the edge and gentle curvature at the bottom. 

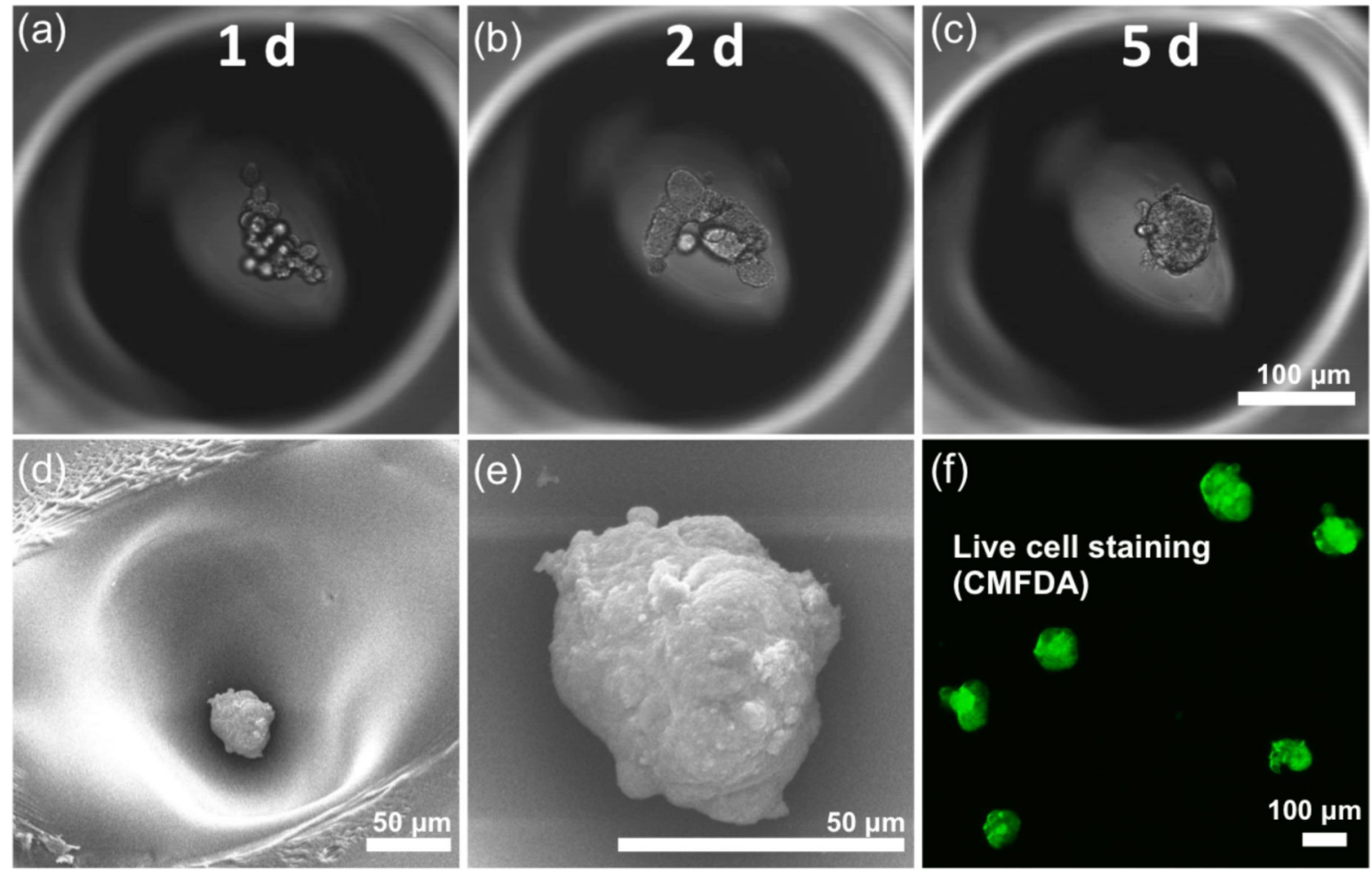

(g)

(h)
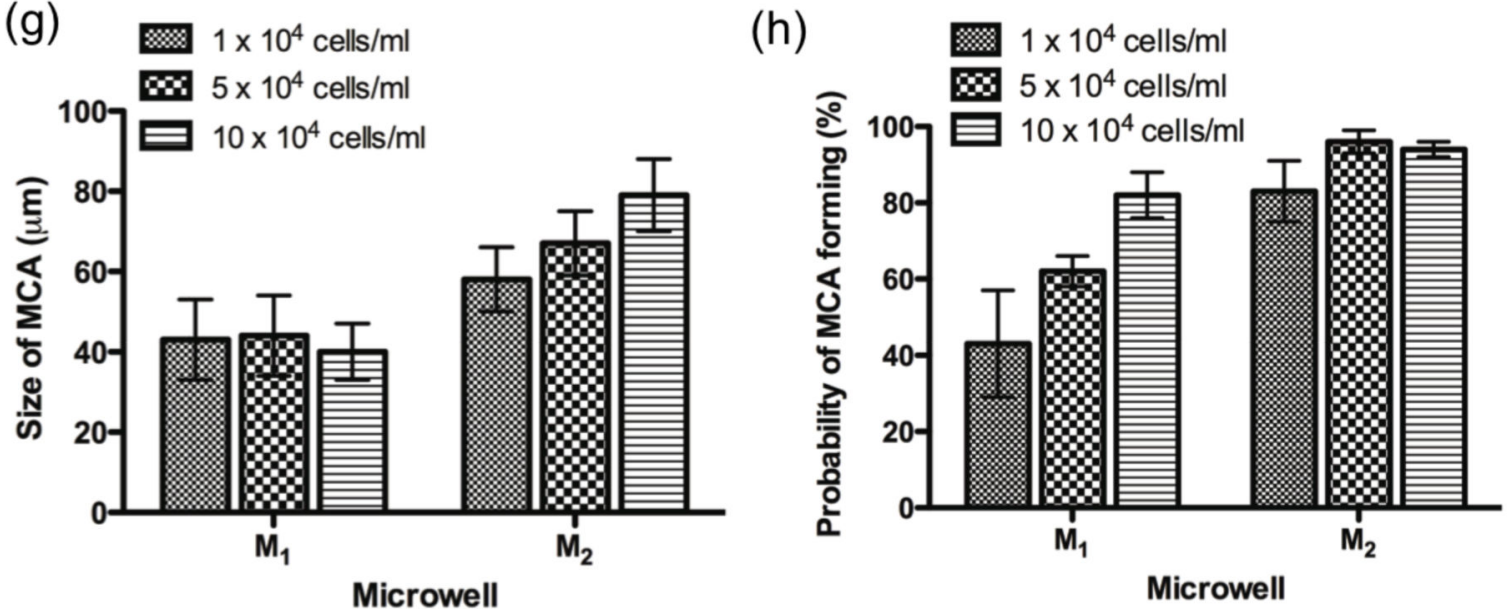

Figure 4.

Generation and characterization of MCAs. (a)-(c) Cell cultures are shown 1, 2, and 5 days after seeding. MCAs formed within 5 d. (d) SEM image illustrating the location of an MCA in a PS microwell. (e) A close-up SEM image of an MCA reveals the surface properties. The majority of cells clumped into a compact aggregate without distinct cell-cell interface indicating maximized intercellular adhesion. (f) CMFDA live cell staining indicated good cell viability and illustrates the shape of the MCAs. (g) Size correlation between MCAs and different cell seeding concentrations in $\mathrm{M}_{1}$ and $\mathrm{M}_{2}$ microwells. (h) Probability of MCA formation across different cell seeding densities in $\mathrm{M}_{1}$ and $\mathrm{M}_{2}$ microwells. 

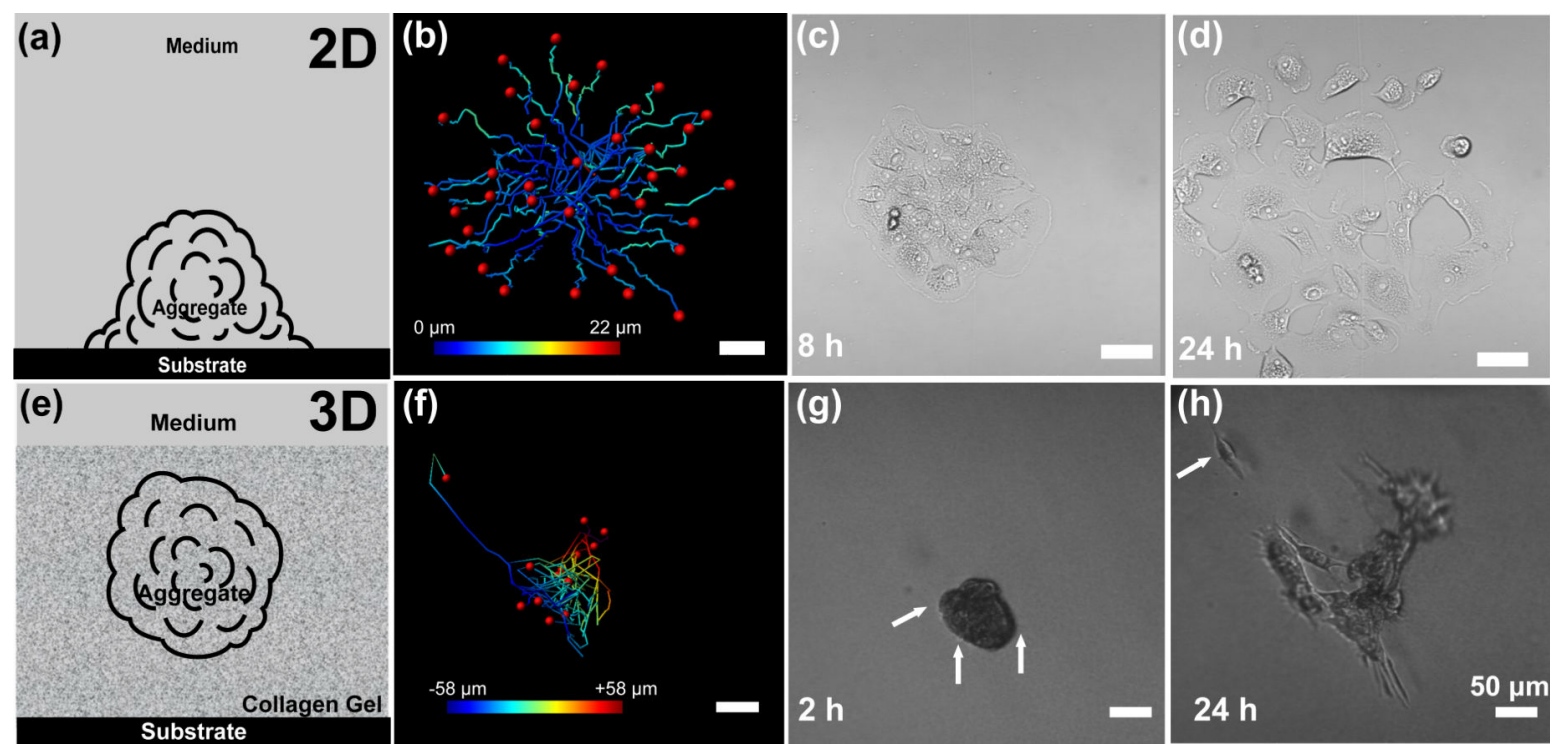

Figure 5.

Schematic diagram and cell migration path over $24 \mathrm{~h}$ in 2D and 3D conditions. (a) Diagram of MCAs in direct contact with the substrate, where dispersion occurs on the 2D surface. (b) Single cells tracking over $24 \mathrm{~h}$ in 2D conditions. (c)-(d) Phase-contrast image of an MCA spreading at 8 and 24 hour in 2D conditions. (e) Diagram of MCAS in 3D suspension in native type I collagen. (f) Cell tracking over $24 \mathrm{~h}$ under 3D conditions. (g) Phase-contrast image of an MCA embedded in 3D collagen matrix showing early formation of pseudopodia at $2 \mathrm{~h}$, indicated by arrows. (h) The same MCA broke up after $24 \mathrm{~h}$, and a single escaped cell was observed (indicated by the arrow). Color bars represent the position along the $\mathrm{Z}$ direction. 

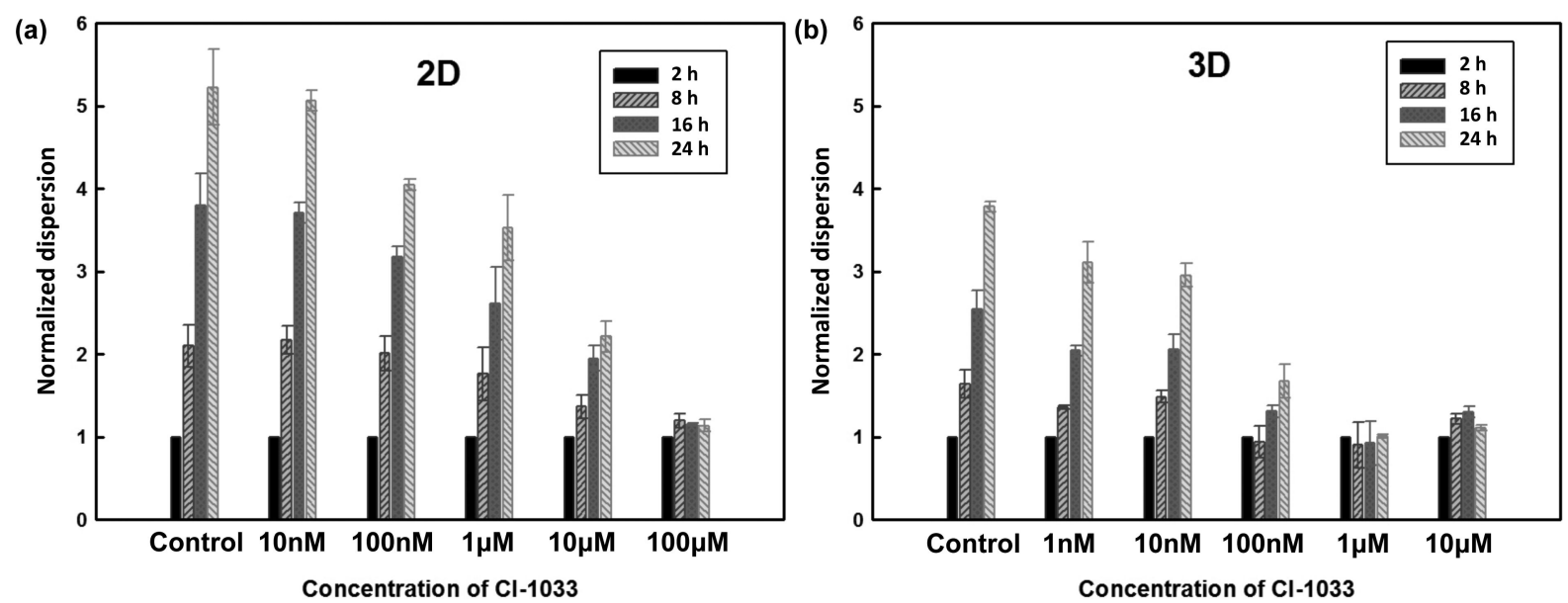

Figure 6.

Influence of CI-1033 on dispersion of A549 MCA in 2D and 3D conditions. Representative samples show normalized dispersion over different concentrations at 2, 8, 16 and $24 \mathrm{~h}$. (a) 2D conditions. (b) 3D conditions. 


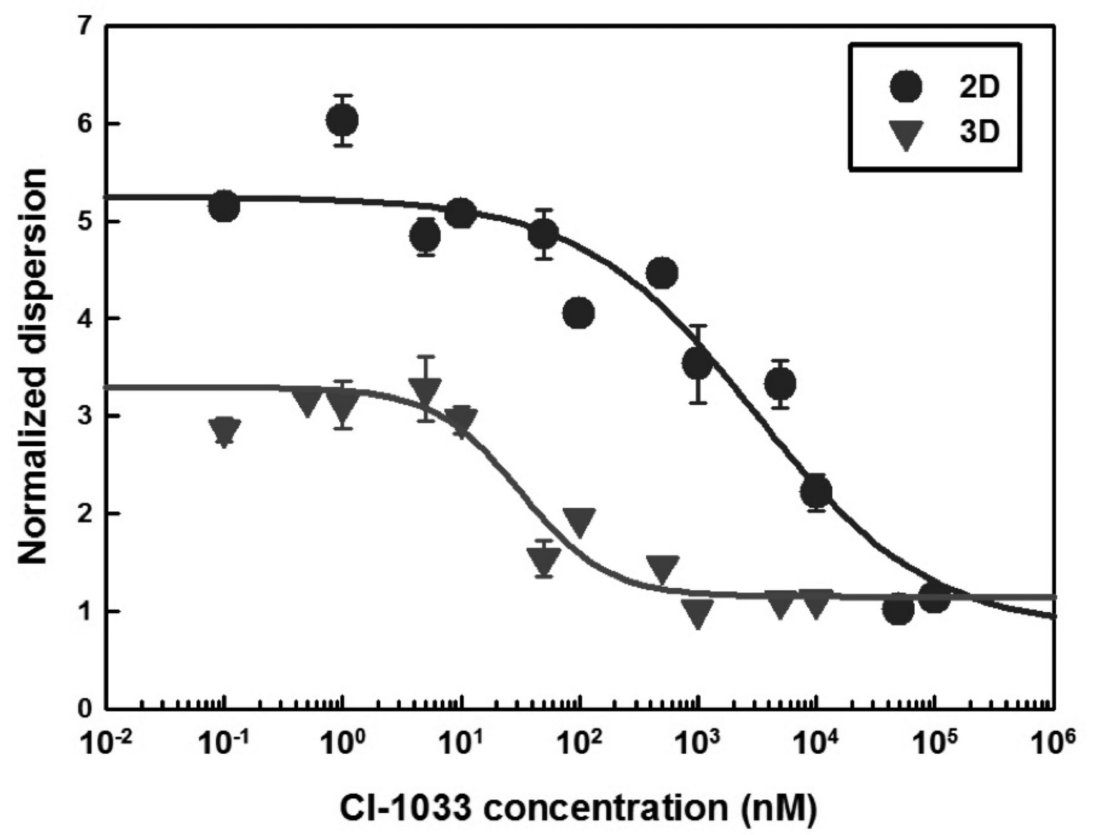

Figure 7.

Dose-response assays on dispersion of A549 MCA after 24 h CI-1033 drug treatment in 2D and $3 \mathrm{D}$ conditions. 